\title{
Generating high-frequency, rotating magnetic fields with low harmonic content
}

\author{
I. Ben-Zvi, X. Chang, V. Litvinenko, W. Meng, A. Pikin, and J. Skaritka \\ Brookhaven National Laboratory, Upton, New York 11973, USA
}

(Received 18 May 2011; published 13 September 2011)

\begin{abstract}
The future electron-ion collider (eRHIC) at Brookhaven National Laboratory demands a high-current, polarized, bunched electron beam [http://www.bnl.gov/cad/eRhic]. One of the challenges here is to combine the bunched beams generated by multiple cathodes so to address the issue of designing and prototyping a combiner with high-frequency $(700 \mathrm{kHz})$ rotational magnetic fields. This article presents its design, and simulation, and details some of the test results from this unprecedented device.
\end{abstract}

DOI: 10.1103/PhysRevSTAB.14.092001

PACS numbers: 29.25.Bx

\section{INTRODUCTION}

The next planned upgrade of Brookhaven's Relativistic Heavy Ion Collider (RHIC) is the future electronRelativistic Heavy Ion Collider (eRHIC) project. Expectedly, the eRHIC will offer, as a high-luminosity, high-energy electron-heavy ion collider or polarizedelectron polarized-proton collider, new scientific opportunities of studying matter. Polarized-electron and polarized-proton collisions demand several conditions: a polarized electron source with high average current ( $\sim 50 \mathrm{~mA})$; a short bunch $(\sim 3 \mathrm{~mm})$; a bunch charge of $3.5 \mathrm{nC}$; a small normalized emittance of about $20 \mu \mathrm{m}$; and an energy spread of $\sim 1 \%$ at $10 \mathrm{MeV}$. The electron bunch's repetition frequency is about $14 \mathrm{MHz}$. The state-of-the-art polarized electron cathode [1] delivers much less than $50 \mathrm{~mA}$; its current is limited by the space charge near the cathode and the quantum efficiency of the polarized source. The source also requires an ultrahigh vacuum environment (better than $10^{-11}$ Torr). Experiments established that, for a given vacuum quality, the product of the current multiplied by the quantum efficiency's lifetime is a constant, usually measured in coulombs [1]. A possible satisfactory approach to achieving a higher current beam is to employ multiple cathodes and combine their multiple bunched beams along the same axis [2]. We decided to follow this method; it led us to developing rotating magnetic fields. Figure 1 is the schematic layout of our concept. Twenty cathodes are spaced evenly along a vertical 30 -cm-diameter circle, and charged to $-200 \mathrm{keV}$. Pulsed laser beams strike these cathodes sequentially at a rotational frequency of $700 \mathrm{kHz}$. This value corresponds to the required $14 \mathrm{MHz}$ bunch-repetition frequency divided by 20, i.e., the number of cathodes. Each bunch is focused by a solenoid and then bent towards the combiner. Since the bunches arrive at the combiner sequentially in a spiral pattern, a rotating magnetic field can kick

Published by the American Physical Society under the terms of the Creative Commons Attribution 3.0 License. Further distribution of this work must maintain attribution to the author(s) and the published article's title, journal citation, and DOI. each one into alignment with the accelerator's axis provided that its rotation phase is synchronized with the arrival time of each bunch. This rotating field is one of the key components in this multiple-cathode gun project.

The direction of electron spin is not affected by the electric field, but will precess about the direction of the magnetic field. To preserve the spin polarization from the cathodes requires that the fixed bending fields after the solenoids and the rotational bending field in the combiner must be either a pair of electric bends or a pair of magnetic ones. We studied both options, and chose the latter scheme because it is a relatively easier and more practical one to establish at the electron's energy level of $200 \mathrm{keV}$.

To preserve the quality of the ultrahigh vacuum, the magnetic combiner must be isolated from it. Meanwhile, the dynamic magnetic field must penetrate the vacuum's wall, as we achieved by using a ceramic vacuum tube. The ceramic tube must have a conductive coating to prevent the buildup of charge on its interior surface that would lead to strong, static space-charge forces. However, at the same time, surface resistance should be sufficiently high to minimize the screening effect of the magnetic field. We accomplished this by ensuring that the conductive coating on the ceramic tube's surface was sufficiently thin. Such coating techniques were demonstrated in many applications.

The system's funneling geometry precluded our adding any focusing component between the fixed bending magnets and the combiner. The space-charge force at our $3.5 \mathrm{nC}$ bunch charge with $200 \mathrm{keV}$ energy is so strong that, when the beams arrive at the combiner, their maximum radius expands from about $5 \mathrm{~mm}$ to about $1.2 \mathrm{~cm}$. Additionally,

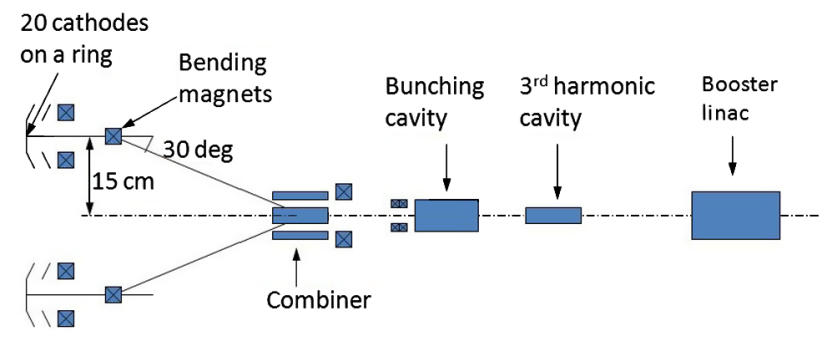

FIG. 1. Schematic layout: "Multiple-cathode gun" system. 
because the beams approaching the rotating deflector are at an angle of $30^{\circ}$, they require a large offset from the combiner's axis at its entrance. These two effects require the combiner to have a large aperture.

Another important issue must be resolved: Because of the geometry of the combining scheme, in the ideal situation, viz., a hard-edged distribution of the magnetic field (the bending field is uniform between the combiner's end surfaces but is zero elsewhere), the beam will lack focusing in the bending direction (the $X$ direction); focusing in the field direction ( $Y$ direction) after bending engenders bunch-tobunch variation of the transverse divergences. If we correct this by adding a rotating quadrupole field downstream of the rotating dipole field, the effective emittance still will increase due to overlapping of the ellipses from bunch to bunch. We found that the best way to solve this problem was to generate rotating dipole and rotating quadrupolemagnetic fields in the same region; then, the beam's profile remains round throughout the bending trajectory.

The injection path deviates from the combiner's axis and is close to the combiner's nonlinear field region, which may increase the emittance.

\section{ROTATOR DESIGN}

A continuous $\cos \theta$ current distribution on a cylindrical surface generates a perfect interior dipole magnetic field. In fact, finite conductors with a discrete quasi- $\cos \theta$ distribution can achieve a fairly pure dipole field within a large good field region as described further down. A similar approach is applicable to the quadrupole-magnetic field generated by a discrete quasi- $\cos 2 \theta$ current distribution. These functions are orthogonal components of the field distribution, and therefore are independent of each other.

Figure 2 illustrates the cross section of the combiner with 12 dipole coils and a ferrite core that enhances field strength. The dipole coils are made of thin-walled hollow copper tubes (return paths are not shown). The time- and angle-dependent currents in these dipole coils are designed to achieve the following values:

$$
I_{D}\left(\Phi_{D}, t\right)=I_{0 D} \cos \left(\omega t+\Phi_{D}\right)
$$

where $I_{O D}$ is the amplitude of the dipole current; $t$ is the time variable; $\omega=2 \pi f$ is the angular frequency of the rotation; and $\Phi_{D}$ is the orientation angle of the dipole coil (with respect to the horizontal). The magnetic field was simulated by OPERA [3], which includes the effects of skin depth and the eddy current. The flux plot shows a uniform dipole field, and Fourier analysis shows that the sextupole component fraction is less than $5 \times 10^{-4}$ within the aperture up to a radius of $6 \mathrm{~cm}$.

The high-permeability ferrite core carries the return flux that significantly reduces the operation currents and the fringe AC noise.

Figure 3 depicts the result of our 3D simulation (OPERA-3D) showing the fringe effect of the field near the

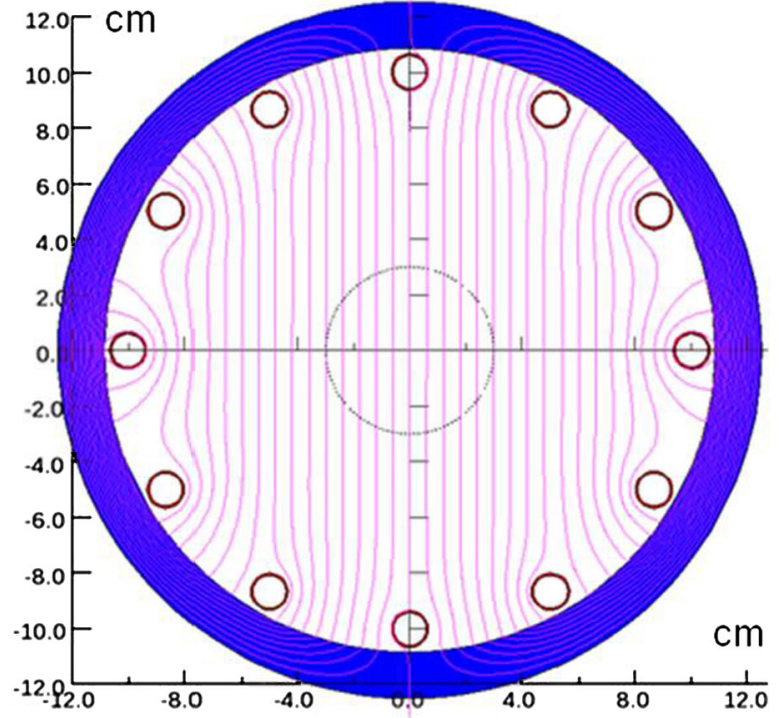

FIG. 2. Plot of flux on the combiner's cross section (with 12 dipole coils and a ferrite core).

ferrite core's end surfaces. The distribution of the bending field resembles a hard-edged box with slightly more extension of the field near the axis than near the ferrite core's edge.

Particle-tracking studies with OPERA-3D models the unequal focusing of the beam in the two transverse directions after bending produced by the combiner without the quadrupole field. The difference is about $1^{\circ}$ at a radius of $1.5 \mathrm{~cm}$, corresponding to an integrated emittance growth of $\sim 3 \mathrm{~mm}$-mrad.

To equalize the focusing of the beam in the two transverse directions during bending, quadrupole coils are wound on the same ferrite core. The currents in the quadrupole coils are

$$
I_{Q}\left(\Phi_{Q}, t\right)=I_{0 Q} \cos 2\left(\omega t+\Phi_{Q}\right),
$$

where $I_{0 Q}$ is the amplitude of quadrupole current; and $\Phi_{Q}$ is the orientation angle of the quadrupole coil with respect

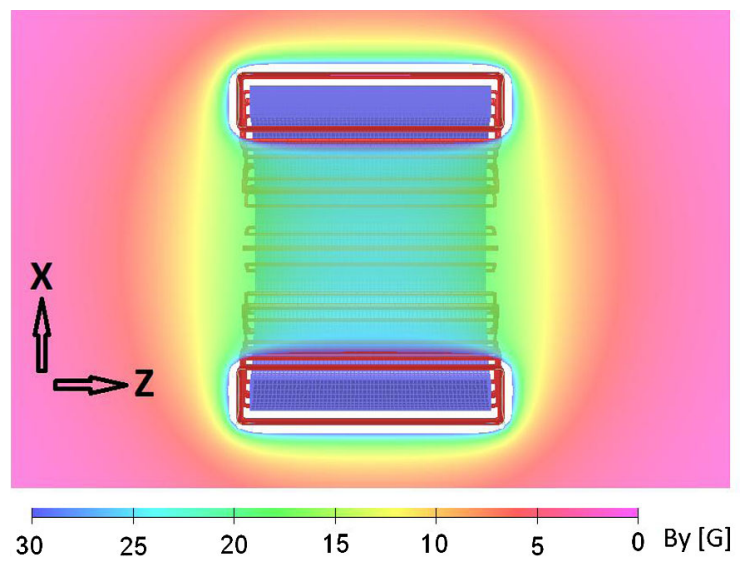

FIG. 3. Bending field on the middle cross section $Z-X$ plane. (The $B_{y}$ contours are not shown inside the ferrite material.) 


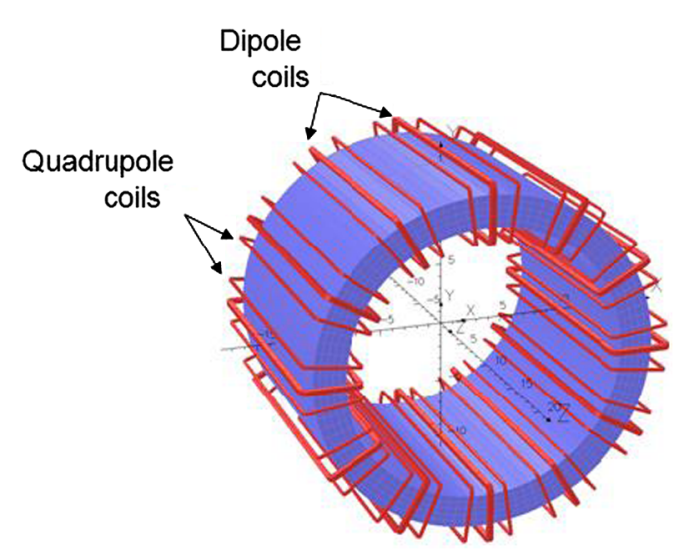

FIG. 4. Combiner with combined dipole and quadrupole fields. The ferrite core is $20 \mathrm{~cm}$ long with an inner radius of $10 \mathrm{~cm}$.

to the horizontal plane. Figure 4 is a perspective view of the ferrite-core combiner with 12 dipole coils and 24 quadrupole coils.

Equations (1) and (2) are functions of time. With advancing time, the dipole and quadrupole fields rotate around the combiner's axis at the frequency $f=700 \mathrm{kHz}$. We confirmed these time-dependent flux patterns via OPERA AC steady-state solutions. The rotation of the fields and multicathode emitted beams are in phase. By adjusting the current in the quadrupole coils, we can optimize the balance in the two focusing directions.

Figure 5 illustrates the trajectories of the $200 \mathrm{keV}$ electrons in the dipole and quadrupole 3D fields as calculated by OPERA. The central field is 26 Gauss, and the integrated field is adjusted to bend the $200 \mathrm{keV}$ electrons by $30^{\circ}$. The amplitude of quadrupole current can be tuned to almost equalize the focusing effect in the horizontal and vertical planes. Then, the emittance is small and the beam's profile is round. Table I compares the different convergence $(+)$ or divergence $(-)$ angles between extreme tracks that resulted from different quadrupole gradients. When the field is a pure dipole field $\left(I_{0 Q}=0\right)$, the parallel beam converges slightly in the $X$ direction

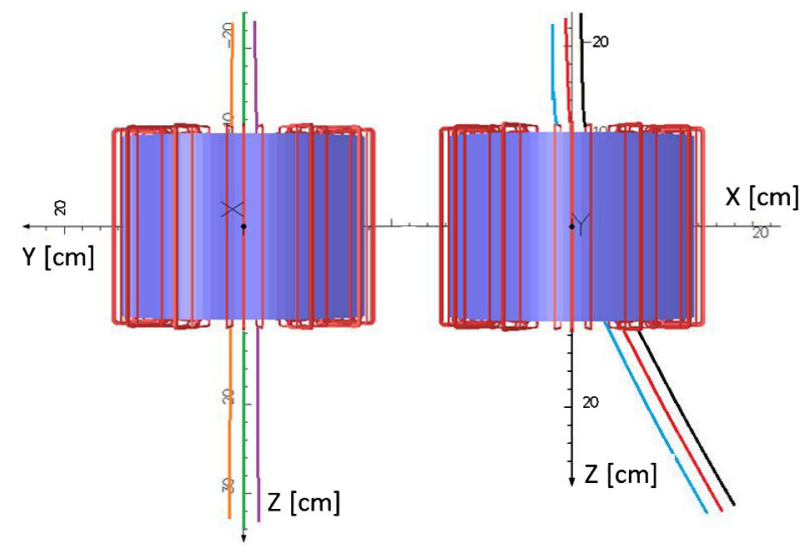

FIG. 5. Electrons' trajectories in the combined dipole and quadrupole fields.
TABLE I. Optimization of quadrupole current.

\begin{tabular}{lcc}
\hline \hline & $\begin{array}{c}\text { Horizontal } \\
\text { converging angle, } \\
\text { m-radians }\end{array}$ & $\begin{array}{c}\text { Vertical } \\
\text { converging angle, } \\
\text { m-radians }\end{array}$ \\
\hline $0 \mathrm{~A}$ & 2.5 & 15.7 \\
$2.4 \mathrm{~A}$ & 8.7 & 9.8 \\
$11.8 \mathrm{~A}$ & 26.0 & -10.0 \\
\hline \hline
\end{tabular}

( $2.5 \mathrm{mrad}$ ) after being bent by the combiner field, This nonzero converging angle in the $X$ direction reflects the distortion of the ideal box-shaped field to a drum-shaped one due to the fringe effect (Fig. 3). The optimum working point giving equivalent focusing in both transverse directions is around $I_{0 Q}=2.4 \mathrm{~A}$.

\section{POWER LOSSES}

With 12 dipole coils, a current amplitude of $I_{0 D}=$ $135.5 \mathrm{~A}$ is required to bend the $200 \mathrm{keV}$ beam by $30^{\circ}$. Calculations show that the total power loss in dipole coils is about $600 \mathrm{~W}$. Water-cooled coils can manage this heat load. The power dissipation will be about the same for 20 dipole coils because the amplitude of current decreases as the number of the coils increases. There is negligible power loss in the quadrupole coils.

The power losses in the ferrite material comprise that from eddy currents, and hysteresis, along with residual loss. In our application, since the peak field is far below saturation, the loss is dominated by eddy currents and residual losses due to the high-frequency excitation. We chose low-loss ferrite MN8CX as the core material; it can operate up to a $\mathrm{MHz}$ range frequency [4]. The peak field in the core is $\sim 620 \mathrm{G}$, and the time-averaged power loss is $648 \mathrm{~W}$, similar to the power loss in the coils. As the ferrite core has a large surface area, the heating power can be dissipated by air cooling. To further lower the heating power from the eddy currents, dividing the core into segments (thickness $\sim 1 \mathrm{~cm}$ ) is recommended [5]. The skin depth of ferrite MN8CX is around 2 to $3 \mathrm{~cm}$ at $700 \mathrm{kHz}$.

It is interesting to compare our $\cos \theta$ design to the Fermilab's beam-sweeping system that demonstrated a rotating field with four conductors [6]. Here, the requirement is to sweep the beam in a circular pattern, whereas our requirement is to maintain the beam's emittance, thus demanding a low harmonic content across a wide aperture. The Fermilab's rotating dipole operates at $625 \mathrm{kHz}$, and produces fields of up to $1.6 \mathrm{kG}$, i.e., higher than needed by the multiple-cathode gun.

\section{PROTOTYPE TESTS}

Each coil is independent of the others, and, in our simulations, we assumed ideal windings. It is important to find a feasible scheme to realize the designed field distribution with minimum expenses, such as via highfrequency power supplies. Certainly, the needed currents 


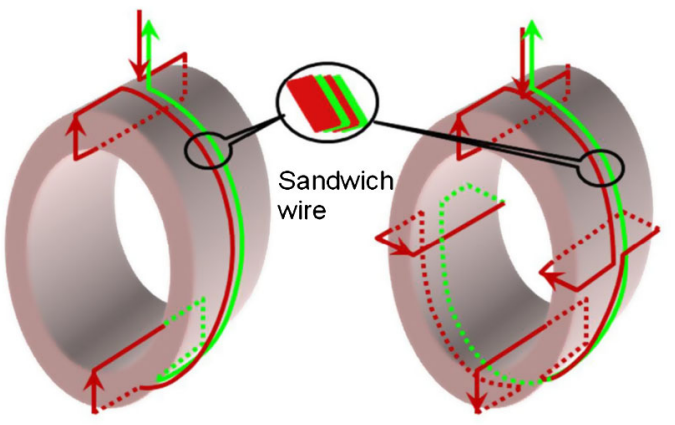

Dipole coil pair winding

Quadruple coil set winding

FIG. 6. Dipole and quadrupole windings.

can be generated by a set of $700 \mathrm{kHz}$ power amplifiers driven by a single oscillator through fixed-phase shifters that produce the right phase shift between the coils. For a 12 conductor dipole winding we need six amplifiers. Other schemes can be considered but are not discussed here as they are not critical to the subject of this paper.

Each dipole coil and its opposite coil have the same amplitude current during operation, due to the dipole field's symmetry, as is shown in Fig. 6. We connected them in serial to form one dipole coil pair, so that, for example, there will be six dipole coil pairs, as in the winding in Fig. 3. Similarly, every four quadrupole coils with the same amplitude current, with $90^{\circ}$ separation, can be connected in serial to form one quadrupole coil set; there will be six quadrupole coil sets in the Fig. 3 winding.

The coils are connected using a multiple-layer sandwichlike wire, as depicted in Fig. 6. This is a variation on the wellknown Litz wire, using flat conductors rather than round ones, which is easier to fabricate. These sandwich wires are located near, but outside, the field-free region in the middle part of the ferrite core. As the net current of the sandwich wire always is zero due to the opposing flows, the sandwich wires are independent. Another advantage of employing the sandwichlike wire is that it reduces dramatically the wire's resistance at high frequency, similar to the filamentous Litz wire. The skin depth of the copper at $700 \mathrm{kHz}$ is $75 \mu \mathrm{m}$. If solid wires are used, then only a small portion of the copper is effective, whereas in the sandwichlike wire with multiple thin conductive layers, the majority of the copper region is utilized effectively for transporting high-frequency current. The power leads for each coil are similarly constructed. With these techniques, we can assure the required current distribution as specified in the simulation.

The phase shift between the dipole coil pairs is achieved by various means that are not discussed in this paper.

To verify our design and analysis, we built a simple combiner prototype and tested it. Figure 7 is a photograph of the prototype: there are four pairs of dipole coil windings and four sets of quadrupole coil windings wound on a ferrite sleeve. The connection wires were twisted wires, instead of the sandwich wire.

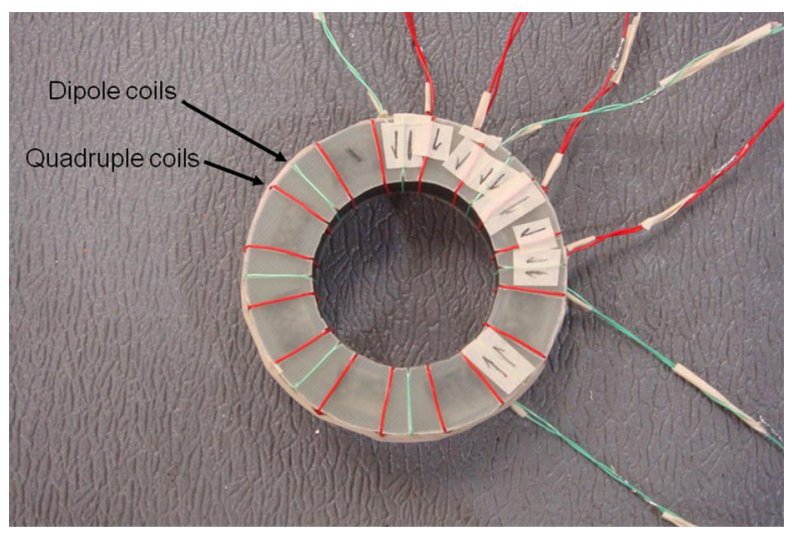

FIG. 7. A combiner prototype with four pairs of dipole coil windings, and four sets of quadrupole coil windings.

First, we measured the coupling between the dipole and the quadrupole mode. We generated the rotating dipole field by applying to the dipole winding signals of $500 \mathrm{kHz}, 2 \mathrm{~V}$ amplitude, and $45^{\circ}$ phase shift between adjacent pairs. The amplitudes of the pickup signal $s$ on the quadrupole coils were all less than $3 \mathrm{mV}$. Second, we similarly generated the rotating quadrupole field, and measured the pickup signal on the dipole windings; we obtained comparable similar results. These tests proved our assumption that the dipole and quadrupole fields are independent each other, and their respective twofold and fourfold symmetries are well preserved.

We also examined the quality of the rotating dipole and quadrupole fields generated by the prototype. They are consistent with our analysis within experimental errors [7].

\section{CONCLUSIONS}

We designed a magnetic funneling device, one of the key components of the multiple-cathode gun for BNL's future eRHIC project. This combiner generates combined dipoleand quadrupole-magnetic fields at a $700-\mathrm{kHz}$ rotating frequency; the symmetries of fields are well preserved, and they can bend and focus the $200 \mathrm{keV}$ beam by $30^{\circ}$ with little emittance growth. Our simulations demonstrate that the radius of the good field (E-3) region is more than $6 \mathrm{~cm}$; the power losses in the coils (including the eddy-current effect) are about $600 \mathrm{~W}$, and the power losses (viz., eddy current, hysteresis, and residual) are about $650 \mathrm{~W}$. These heat losses are easily dissipated with water cooling, even possibly by air cooling. With our simple prototype, we verified the complete independence of the device's dipole field and the quadrupole field.

[1] P. Adderley et al., in Proceedings of the 2011 Particle Accelerator Conference, New York, 2011, WEODS3 (to be published).

[2] X. Chang et al., "A Multiple Cathode Gun Design for the eRHIC Polarized Electron Source," in Proceedings of 
the 2011 Particle Accelerator Conference, New York, 2011, WEP263 (to be published).

[3] Vector Fields Limited, England.

[4] http://www.cmi-ferrite.com.

[5] G. E. Schaller (private communication).

[6] F. M. Bieniosek, O. Kurnaev, A. Cherepakhin, J. Bielicki, and J. Dinkel, in Proceedings of the 18th Particle
Accelerator Conference, New York, 1999 (IEEE, New York, 1999).

[7] X. Chang et al., "Rotating Dipole and Quadrupole Field for a Multiple Cathode System," in Proceedings of the 2011 Particle Accelerator Conference, New York, 2011, TUP147 (to be published). 